\title{
MASCC/ESMO/EAPC survey of palliative programs
}

\author{
Mellar P. Davis • Florian Strasser • Nathan Cherny • \\ Norman Levan
}

Received: 25 September 2014 / Accepted: 30 November 2014/Published online: 12 December 2014

(C) Springer-Verlag Berlin Heidelberg 2014

\begin{abstract}
Background Palliative care program structure is important to integrating palliative services into cancer care. A first step in understanding the structure of palliative care programs is to survey existing programs.

Method This data was generated from members of MASCC, the European Society of Medical Oncology (ESMO), and the European Association of Palliative Care (EAPC) who completed the surveys on the website. A survey questionnaire was developed using the survey tool developed by Dr. Hui and colleagues by permission which was modified for the purposes of this study. Findings were described in number and percentages. Inferential statistics involved the Fisher's exact test for factors with two levels, chi-Square test for unordered categorical factors with greater than two levels, CochranArmitage trend test for ordered categorical factors, and the Wilcoxon rank sum test for measured factors.

Results Sixty-two program leaders completed the survey. Most programs had been in existence greater than 5 years and were led by oncology trained physicians who had an
\end{abstract}

\section{P. Davis $(\square)$}

Cleveland Clinic Lerner School of Medicine Case Western Reserve

University, Cleveland, OH, USA

e-mail: davism6@ccf.org

M. P. Davis

Palliative Medicine and Supportive Oncology Services, Division of Solid Tumor, Taussig Cancer Institute, The Cleveland Clinic,

Cleveland, OH, USA

\section{F. Strasser}

Department Internal Medicine \& Centre for Palliative Care, Oncological Palliative Medicine, Clinic for Oncology and Hematology, Cantonal Hospital St. Gallen, Gallen, Switzerland

N. Cherny $\cdot$ N. Levan

Department Medical Oncology, Shaare Zedek Medical Center, Jerusalem, Israel additional specialty. Most programs had consultative services and outpatient clinics with fewer having inpatient beds and institutionally associated hospices. Most programs provided patient continuity. Patients were generally seen late in the course of illness with the average survival of 23 days when seen as inpatients and 40 days when seen as outpatients. Less than half had palliative care fellowship training programs. Most had research structures in place.

Discussion These findings differ from results reported in a previous survey which may reflect a European palliative care program structure. However, there were similarities which include a high inpatient palliative care unit mortality and short survival of patients seen as outpatients, indicating that referrals to palliative care occur late in the course of cancer.

Conclusions This study not only differs in some respects to a previous survey of palliative care programs but also confirms the late referral of patients to palliative care.

Keywords Palliative $\cdot$ Inpatient $\cdot$ Consultative $\cdot$ Mortality Service

\section{Introduction}

Integration of palliative care into cancer care is highly endorsed by the World Health Organization, the Institute of Medicine, the European Society of Medical Oncology, and the American Society of Clinical Oncology [1, 2]. Palliative Care has increasing relevance for the care of advanced cancer patients $[3,4]$. By improving quality of life with aggressive symptom management, reducing the cost of care, and possibly improving survival, palliative care can no longer be confused with care at the end of life [5-7].

Elements of palliative care include standard definitions, component parts, models of care delivery, standardized assessment tools, educational programs, fellowship training, and 
accreditation. Very little is known about the structure of palliative care programs internationally. In light of this, the Multinational Association of Supportive Care on Cancer (MASCC), the European Society of Medical Oncology (ESMO), and the European Association of Palliative Care (EAPC) surveyed palliative programs through each society's membership.

\section{Methods}

Research design

This study used an analysis from a cross-sectional survey conducted through the MASCC website using Survey Monkey (www.surveymonkey.com/). The survey was in two parts and involved palliative care program leaders (Fig. 1). The first part centered on palliative care integration into oncologic care and is reported separately. This project was reviewed by the Cleveland Clinic Institutional Review Board and was granted exemption from consent. The main method of this survey was a questionnaire which was modified from the original questionnaire by Dr. Hui and colleagues [8].

Sample

This data was generated from members of MASCC, ESMO, and the EAPC, who completed the survey on the website. Members were informed of the survey and invited to complete the survey by entering the MASCC website. Repeated invitations were made until no further surveys were received. The survey was sent November 15, 2011, to 676 MASCC members and made available on the MASCC website for nonMASCC members. The survey was removed from the website April 20, 2012.

\section{Questionnaire}

For the purposes of this study, a survey questionnaire was designed using a modified version of the survey tool developed by Dr. Hui and colleagues with their permission [8]. Modifications involved a question about designated ESMO

\section{Survey for Palliative Care Program Leaders}

Thank you for participating in this survey. Please try to complete the following survey in one sitting. It should take no more than 15 minutes. When answering questions regarding numbers or percentages, please use approximations (i.e. we do not expect you to look up the information in detail). Thank you!

I. Palliative Medicine Services

A. Does your institution provide any palliative care services?

$\square$ Yes (please complete this section)

$\square$ No (please skip to Section V)

B. What is the name of your palliative care program? (Check all that apply)

$\square$ Comprehensive cancer care

$\square$ Pain and symptom management

$\square$ Palliative care

प Supportive care

$\square$ Other:

C. How long approximately has your palliative care program been officially seeing patients?

$$
\square<1 \text { year } \quad \square \text { 1-2 years } \square 3-5 \text { years } \quad \square>5 \text { years }
$$

D. What are the specific kinds of palliative care services that are available? (Check all that apply)

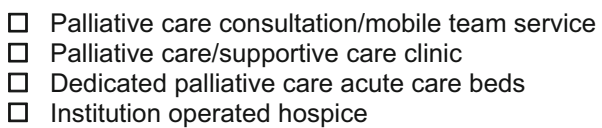

E. What are the type(s) of services that your palliative care team offers? (Check all that apply) $\square$ Assessment and management of psychiatric disorders

Assistance in obtaining advance directives and Do Not Resuscitate (DNR)

$\square$ Assistance in resolving complicated ethical issues

$\square$ Assistance with referrals to hospice, home care, or other placements

$\square$ Comprehensive care plan for those requiring comfort care

$\square$ Pain consultation

$\square$ Psychosocial support

$\square$ Symptom management

F. Approximately, what proportion of patients seen by palliative care belong to the pediatric age group ( $<18$ year old)?
$\square 0 \%$
- $1-25 \%$
$\square 26-50 \%$
$\square 51-75 \%$
$\neg 76-100 \%$

Fig. 1 Palliative care leadership survey 
I. Palliative Medicine Services (continued)

G. What is the professional background of the palliative care program leader? (Check all that apply)

$\square$ Anesthesia
$\square$ Family medicine
$\square$ Internal medicine
$\square$ Medical Oncology

Nursing

Palliative medicine

Pediatrics

Psychiatry

$\mathrm{H}$. Within your program, please indicate the approximate number of paid personnel assigned to palliative care:

\begin{tabular}{|l|c|c|c|c|c|c|}
\hline Personnel & 0 & $1-5$ & $6-10$ & $11-25$ & $26-50$ & $>50$ \\
\hline Ward nurses (inpatient) & & & & & & \\
\hline Clinic nurses (outpatient) & & & & & & \\
\hline
\end{tabular}

\begin{tabular}{|l|l|l|l|l|l|}
\hline Personnel & $\mathbf{0}$ & $\mathbf{1}$ & $\mathbf{2 - 5}$ & $\mathbf{6 - 1 0}$ & $\mathbf{> 1 0}$ \\
\hline Chaplains & & & & & \\
\hline Dieticians & & & & & \\
\hline $\begin{array}{l}\text { Mid-level providers } \\
\text { (advanced nurse } \\
\text { practitioners or physician } \\
\text { assistants) }\end{array}$ & & & & & \\
\hline Palliative care physicians & & & & & \\
\hline Rehab (PT/OT) & & & & & \\
\hline Pharmacists & & & & & \\
\hline Psychiatrists & & & & & \\
\hline Psychologists & & & & & \\
\hline Social workers & & & & & \\
\hline Other & & & & & \\
\hline
\end{tabular}

I. Approximately, how many full-time equivalent (FTE) physician positions are available in your palliative care program?

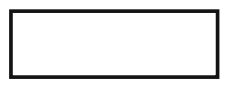

J. Approximately, how many physicians on your palliative care team have at least $20 \%$ academic protected time?

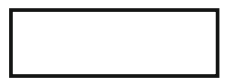

K. Does your palliative care program require physicians to be certified (finished a fellowship and taken boards)?

$$
\square \text { Yes } \quad \square \text { No }
$$

L. Does your palliative care program require nurses to be certified (taken boards in palliative nursing)?

$$
\square \text { Yes } \quad \square \text { No }
$$

Fig. 1 (continued)

centers. Exemption from written or verbal informed consent was obtained from the Institutional Review Board of the Cleveland Clinic. Palliative Care program leaders entered the MASCC website to complete the survey using Survey Monkey (www.surveymonkey.com/). The questionnaire queried palliative care program leaders on the name of the program, how long the program was in existence, and the type of services provided (Fig. 1). Leaders were asked if their care included pediatric patients. Questions involved professional backgrounds of the program leader, number of full-time equivalence, certification and patient continuity. In regards to inpatient palliative care, leaders were asked about inpatient palliative care beds, duration of stay in hospital, number of inpatient discharges per month, median survival after admission, reasons for admission, number of family conferences, and advanced directives. Questions regarding consultative services involved the presence of a consultative service, availability, number of referrals per month, survival, and referral sources. Regarding outpatient care, leaders were asked about outpatient clinics, number of days per week a clinic is held, referrals per month, and survival. A number of other questions were asked about institution-operated hospices, education services, fellowship programs, mandatory palliative care rotations for oncology fellows and other trainees, grand rounds, length of training for fellows, accreditation requirements, research and palliative care funding. Responses are reported for all leaders combined and broken 


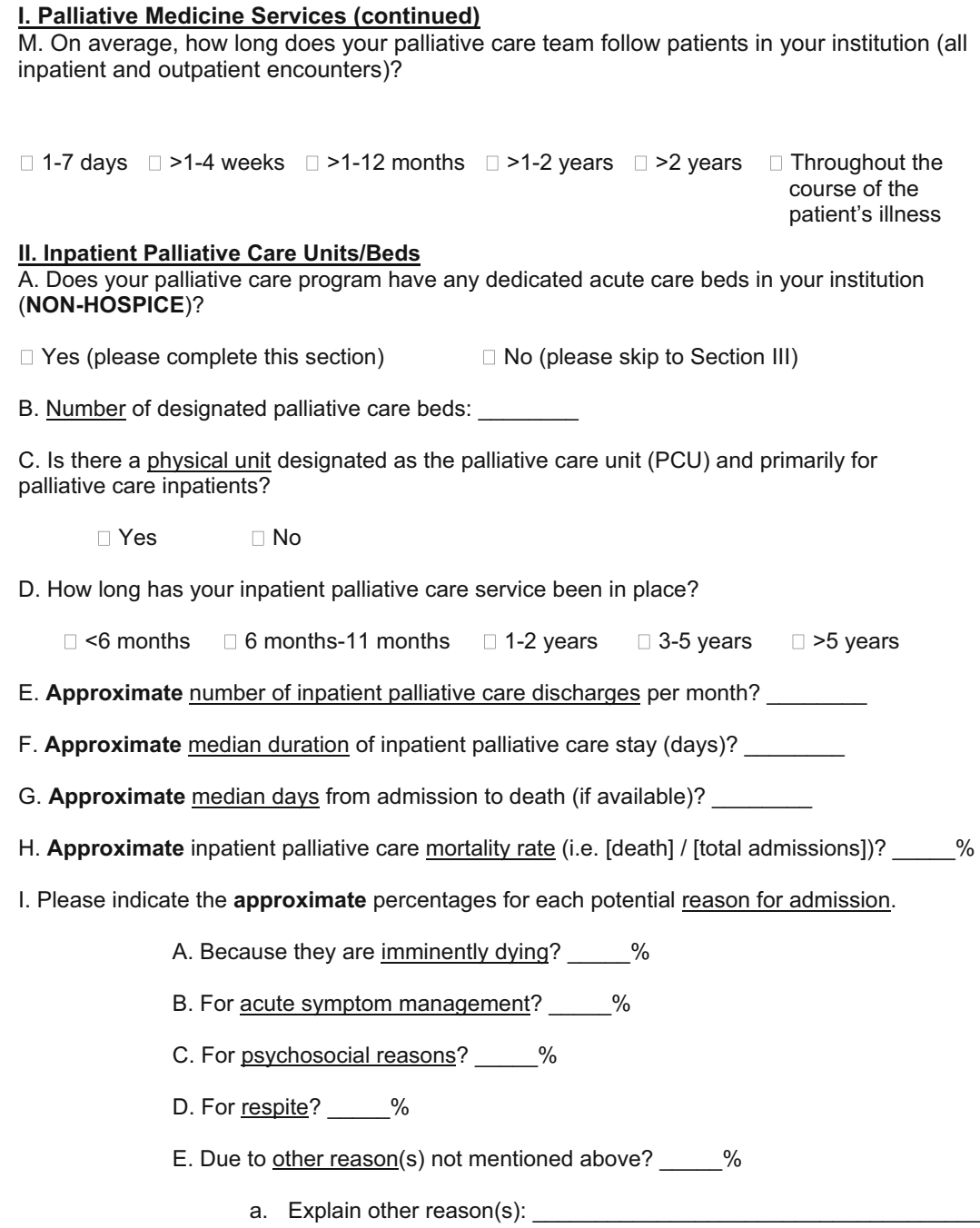

Fig. 1 (continued)

down by whether the program is relatively new (less than 5 years old) or mature (greater than 5 years old).

\section{Statistics}

Findings were described in number and percentages. Inferential statistics involved the Fisher's exact test for factors with two levels, chi-square test for unordered categorical factors with greater than two levels, Cochran-Armitage trend test for ordered categorical factors and the Wilcoxon rank sum test for measured factors. A $p<0.05$ was considered statistically significant.

\section{Results}

Sixty-two program leaders completed the survey (Table 1). One responder indicated that his or her institution did not provide palliative care services and was therefore excluded from analysis. Program names were often described using a single phrase. Thirty-six percent (22/61) used a single descriptor "Palliative Care", $39 \%$ were described in two or more phrases (Table 1). The majority of the palliative programs completing the survey were $>5$ years $(43 / 61,70 \%), 3(5 \%)$ were $<1$ year, 4 (7\%) 1-2 years, and 11 (18\%) 3-5 years old. Forty-nine ( $80 \%$ ) of programs had mobile/consultative teams, $80(82 \%)$ has palliative care/supportive clinics, and $38(62 \%)$ has inpatient beds dedicated to palliative care. Only half (30 or $49 \%$ ) had all three services and only $25 \%$ (15) had an institutionally operated hospice. There was a trend for older programs to have outpatient clinics $(p=0.09)$. Types of services offered included assessment and management of psychiatric disorders, discussions regarding advanced directives, resulting ethical complex issues, assisting with hospice referral, developing comprehensive care plans, pain and symptom management, and psychosocial support. Most programs (59\%) offered seven to eight services. Approximately $1 / 3$ of programs cared for pediatric patients. Approximately half 
II. Inpatient Palliative Care Units/Beds (continued)

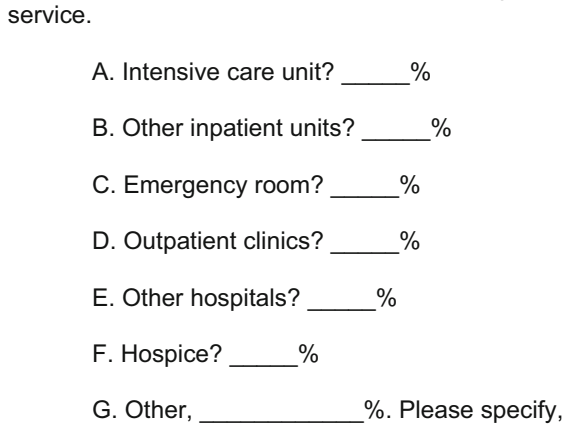

K. Approximately, what proportion of patients receives regular psychosocial assessments on each admission?

$$
\square 0 \% \quad \square 1-25 \% \quad \square 26-50 \% \quad \square 51-75 \% \quad \square 76-100 \%
$$

L. Approximately, what proportion of inpatients has family conferences during admissions?

$\square 0 \% \quad \square 1-25 \% \quad \square 26-50 \% \quad \square 51-75 \% \quad \square 76-100 \%$

M. Approximately, how often do primary oncologists attend these family conferences?

$$
\square 0 \% \quad \square 1-25 \% \quad \square 26-50 \% \quad \square 51-75 \% \quad \square 76-100 \%
$$

N. Approximately, what proportion of inpatients has Do-Not-Resuscitate orders?

$$
\square 0 \% \quad \square 1-25 \% \quad \square 26-50 \% \quad \square 51-75 \% \quad \square 76-99 \% \quad \square 100 \%
$$

\section{Consultation Service}

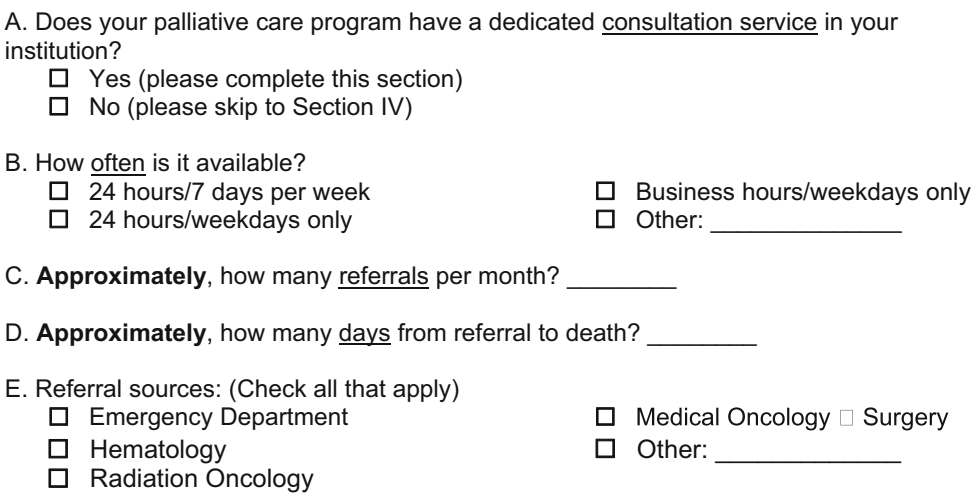

Fig. 1 (continued)

(48 \%) had $>5$ inpatient nurses dedicated to palliative care; $10 \%$ had $>5$ outpatient clinic nurses dedicated to palliative care.

Most palliative care program leaders who completed the survey were oncologists (74\%) though $69 \%$ of respondents recorded more than one specialty. The majority of programs have a chaplain, dietitian, mid-level provider, rehabilitation personal, psychologist, and social worker dedicated to palliative care. Less than half (27 of 62 programs, $46 \%$ ) had a dedicated pharmacist. The median number of full-time equivalent physicians was 2 (range $0-15$ ) with half (55\%) of programs providing physicians with $20 \%$ academic protected time. Thirty-five $(58 \%)$ programs required physician certification in palliative care or to complete a palliative care fellowship; $47 \%(28 / 62)$ of program required nurse certification in palliative care.

Most programs (75\%) provided continuity (followed patients for greater than 4 weeks or throughout the course of disease). Seventy-four percent (43) had dedicated acute care beds within a unit with the median number of 10 beds (range 0-43), median length of stay was 10 days (range 3-98), and median discharges per month was 24 (range 2-250). The primary reason for admission to the inpatient palliative unit was symptom management $(60 \%)$. Admission for reason of imminent death was $10 \%$. However, the median inpatient palliative care mortality rate was $40 \%$ (range $2-99 \%$ ). The 
IV. Palliative Care Outpatient Clinic

A. Does your palliative care program see patients in the outpatient setting? (Check all that apply)

$\square$ Yes, we have a dedicated palliative care outpatient clinic (please complete this section)

$\square$ Yes, we see patients in oncology clinics (please complete this section)

No (please skip to Section V)

B. Approximately, how many days per week is it held?

C. Approximately, how many referrals per month?

D. Approximate number of days from referral to death (if available)?

E. Referral sources: (Check all that apply)

$\square$ Emergency Department

$\square$ Medical Oncology

$\square$ Surgery

$\square$ Hematology

$\square$ Radiation Oncology

$\square$ Other:

V. Hospice

A. Does your institution operate a hospice? $\square$ Yes $\square$ No

If yes, please indicate the approximate average hospice daily census:

\section{Vl. Educational Services Targeting Palliative Care}

A. Fellowship program for Palliative Medicine? $\square$ Yes $\square$ No

If yes, approximate number of clinical fellows per year:

If yes, approximate number of research fellows per year:

B. Mandatory palliative care rotations for...

$\begin{array}{llrl}\text { Medical oncology/hematology fellows } & \square \text { Yes } & \square \text { No } & \square \text { Not applicable } \\ \text { Radiation oncology fellows } & \square \text { Yes } & \square \text { No } & \square \text { Not applicable } \\ \text { Pediatric oncology fellows } & \square \text { Yes } & \square \text { No } & \square \text { Not applicable } \\ \text { Medical students } & \square \text { Yes } & \square \text { No } & \square \text { Not applicable } \\ \text { Other residents or fellows: } & \square \text { Yes } & \square \text { No } & \text { Not applicable }\end{array}$

C. Training of mid-level providers in palliative care: $\square$ Yes $\square$ No

D. Dedicated palliative care grand rounds: $\square$ Yes $\square$ No

If yes, how many times per week: $\quad \square 1 \quad \square 2 \quad \square 3$ or more

E. Length of training for fellows for certification:

$\square 1$ Year

$\square 2$ Years

3 Years

$\square$ Other

F. Are there palliative board examinations? $\square$ Yes $\square$ No

G. Are there recognized accreditation requirements to be recognized as a specialist in palliative medicine? $\quad \square$ Yes $\square$ No

Fig. 1 (continued

median number of days from admission to death was 10.5 (range 3-55 days). Major sources of referral to the inpatient unit were transfers from non-ICU acute care beds $(20 \%)$, emergency departments (10\%), and outpatient clinics (20\%), and only $5 \%$ coming from intensive care units. In half of programs more than $75 \%$ of patients in the hospital on palliative units had psychosocial assessments during admission, had family conferences during the inpatient stay, and most had standing DNR orders. However, only $36 \%$ of programs had the oncologist attend $>75 \%$ of family meetings, while $45 \%$ of programs $(N=19)$ stated that the oncologist attended $15 \%$ or fewer family conferences. Although palliative inpatient consultative services were uniformly provided, most $(57 \%)$ were not $24 \mathrm{~h} / 7$ days a week services. On average, services had 25 referrals per month (range 3-400), and most sources of referral were medical and radiation oncology and surgery. The median time from inpatient consultation to death was 23 days (range 7-97). 
VII. Research in Palliative Care

A. Is there a research program in palliative care?

Yes (please complete this section)

$\square$ No (End of survey. Thank you)

B. The research team consists of... (Check all that apply)

$\begin{array}{lll}\square \text { Chaplains } & \square \text { Data analysts } & \square \text { Psychologists } \\ \square \text { Pharmacists } & \square \text { Physicians } & \square \text { Social workers } \\ \square \text { Rehab therapists } & \square \text { Research nurses } & \\ \text { (PT/OT) } & \square \text { Dieticians } & \end{array}$

C. Outside funding:

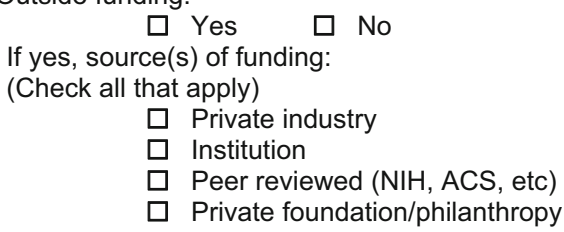

VII. Research in Palliative Care (continued)

D. Please indicate the types of palliative care studies conducted over the last year: (Check all that apply)

$\square$ Prospective randomized therapeutic trials

$\square$ Prospective randomized non-therapeutic trials

$\square$ Other prospective non-randomized studies

$\square$ Retrospective studies

$\square$ Case series/case reports

$\square$ Qualitative studies

$\square$ Others. Please specify,

$\square$ None

E. Please indicate the approximate number of abstracts presented at meetings by your department last year:

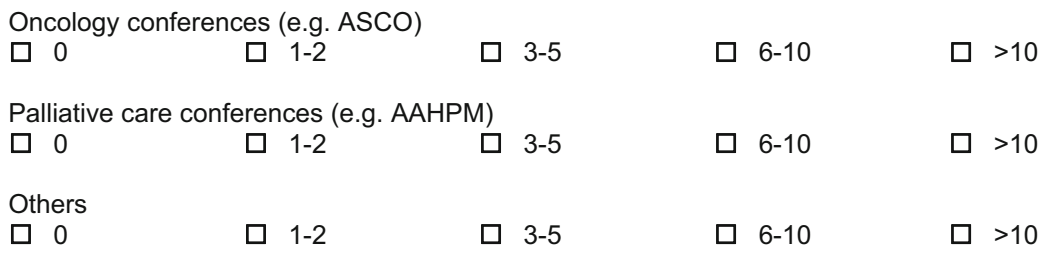

F. Please indicate the approximate number of papers published by your department last year:

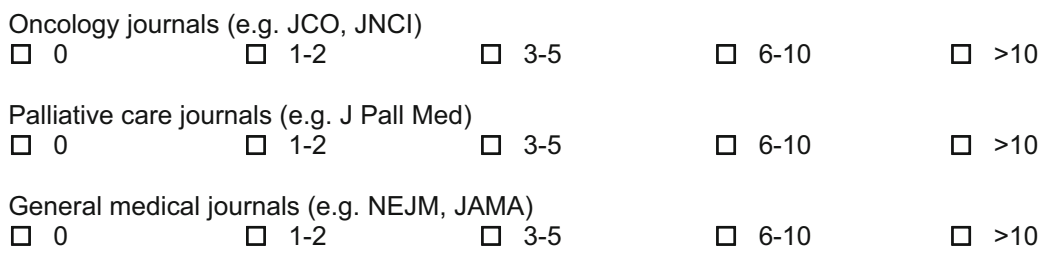

Fig. 1 (continued)

Outpatient clinics were held a median of 5 days/week (range 0.5-7) and saw a median of 30 new referrals per month (range 3-250). Most referrals were from medical and radiation oncology. Approximately half $(45 \%)$ had dedicated palliative care outpatient clinics only, $19 \%$ saw patients in oncology clinics only and an additional $19 \%$ saw patients both in palliative care outpatient clinics and oncology clinics.
Approximately $1 / 3$ of palliative care programs (37\%) had active fellowship training, half (52\%) had one to two clinical fellows per year and half (48 \%) had more than two clinical fellows in their program. Length of training for fellows to be certified was less than 1 year for $67 \%$ of programs. Half of programs $(56 \%)$ had one to two research fellows per year. Excluding programs without ties to cancer care, half (56\%, 27/48)) of programs provided palliative care rotations for 


\section{Integration}

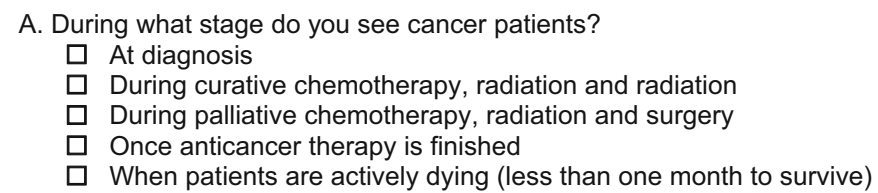

B. Do members of the palliative care department attend (nurses, physicians)?

$\square$ Oncology conferences

$\square$ Oncology tumor boards

$\square$ Oncology administrative meetings

$\square$ Oncology outpatient or inpatient services

C. Do members of the oncology department attend (nurses, physicians)?

$\square$ Palliative medicine lectures

$\square$ Palliative medicine administrative meetings

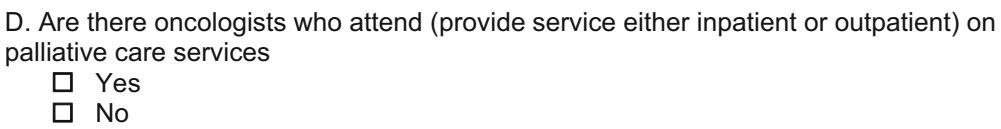

E. Are there palliative specialists who attend on oncology services (either outpatient or inpatient?)

$\square$ Yes

$\square$ No

F. Is there any integration of the palliative care program with other departments?
$\square$ Intensive Care
$\square$ Geriatrics
$\square$ Cardiology
$\square$ Neurology
$\square$ Pulmonology
$\square$ Pediatrics
$\square$ Pain medicine
$\square$ Rehabilitation
$\square$ Other

Comments:

Thank you for your participation!

Fig. 1 (continued)

oncology fellows, $33 \%$ (14/43) for radiation oncology fellows, $51 \%$ (23/45) for other fellows/residents, $35 \%(15 / 43)$ for medical students, $9 \%(3 / 31)$ for pediatric oncology fellows, and $61 \%(34 / 56)$ trained mid-level providers. Half of programs incorporated Grand Rounds at least once per week $(68 \%)$ or two to three times per week (32\%).

Approximately $2 / 3$ of programs (64 \%) had research programs, usually consisting of a physician $(100 \%)$, data analyst $(75 \%)$, research nurse $(72 \%)$, and psychologist (56\%). Less than half of these programs (44\%) were fully staffed and almost $(62 \%)$ had outside funding, usually from private foundations and philanthropy. Most research programs conducted prospective trials ( $86 \%$ ); half were involved in retrospective studies, case reports/series, and qualitative studies. For the most part, research programs reported their findings in palliative care and oncology journals. Seventy percent of these had one publication in a palliative care journal over the last year, $68 \%$ had at least one publication in an oncology journal, and $49 \%$ had at least one in a general medical journal.

The number of young palliative care programs was small so results need to be taken with caution when compared to more mature programs. More mature programs had shorter hospital stays (median of 9.5; range 3-96) versus younger programs (median 14.5; range 9-98; $p=0.007$ ). Mature programs had a greater number of referrals (median 30, range 3-400) versus younger programs (median 15 ; range $4-40 ; p=0.04$ ). Mature programs provided palliative care rotations for non-oncology fellows and residents more frequently (60 vs. $20 \%$; $p=0.04$ ).

\section{Discussion}

A picture emerges from this survey which characterizes palliative care programs. The average program has been in 
Table 1 Survey results

\begin{tabular}{|c|c|c|c|c|c|}
\hline Group & Question & $\begin{array}{l}\text { All } \\
\text { leaders } \\
\text { Combined }\end{array}$ & $\begin{array}{l}\text { Programs } \\
\leq 5 \text { years } \\
\text { old } \\
(n=17)\end{array}$ & $\begin{array}{l}\text { Programs } \\
>5 \text { years old } \\
(n=44)\end{array}$ & $p^{\mathrm{a}}$ \\
\hline \multirow[t]{6}{*}{ All programs } & Q4: Specific palliative care (PC) services offered & & & & \\
\hline & PC consultation/mobile team service & $49(80 \%)$ & $12(71 \%)$ & $37(84 \%)$ & 0.29 \\
\hline & PC/supportive care clinic & $50(82 \%)$ & $11(65 \%)$ & $39(89 \%)$ & 0.06 \\
\hline & Dedicated PC acute care beds & $38(62 \%)$ & $8(47 \%)$ & $30(68 \%)$ & 0.15 \\
\hline & Institution operated hospice & $15(25 \%)$ & $3(18 \%)$ & $12(27 \%)$ & 0.52 \\
\hline & All of the first 3 services & $30(49 \%)$ & $5(29 \%)$ & $25(57 \%)$ & 0.09 \\
\hline \multirow[t]{10}{*}{ All programs } & Q5: Types of services offered & & & & \\
\hline & Assessment/management of psych. Disorders & $32(52 \%)$ & $7(41 \%)$ & $25(57 \%)$ & 0.39 \\
\hline & Assistance in obtaining advance directives & $37(61 \%)$ & $8(47 \%)$ & $29(66 \%)$ & 0.24 \\
\hline & Assistance in resolving complex ethical issues & $38(62 \%)$ & $9(53 \%)$ & $29(66 \%)$ & 0.39 \\
\hline & Assistance with referrals to hospice, etc. & $51(84 \%)$ & $15(88 \%)$ & $36(82 \%)$ & 0.71 \\
\hline & Comprehensive care plan & $51(84 \%)$ & $14(82 \%)$ & $37(84 \%)$ & 1.0 \\
\hline & Pain consultation & $58(95 \%)$ & $16(94 \%)$ & $42(95 \%)$ & 1.0 \\
\hline & Psychosocial support & $54(89 \%)$ & $15(88 \%)$ & $39(89 \%)$ & 1.0 \\
\hline & Symptom management & $60(98 \%)$ & $17(100 \%)$ & $43(98 \%)$ & 1.0 \\
\hline & $7-8$ services & $36(59 \%)$ & $8(47 \%)$ & $28(64 \%)$ & 0.26 \\
\hline \multirow[t]{3}{*}{ All programs } & Q6: \% pediatrics & & & & \\
\hline & 0 & $39(66 \%)$ & $14(88 \%)$ & $25(58 \%)$ & \\
\hline & $>0^{\mathrm{b}}$ & $20(34 \%)$ & $2(12 \%)$ & $18(42 \%)$ & 0.06 \\
\hline \multirow[t]{13}{*}{ All programs } & Q7: Professional background of program leaders & & & & \\
\hline & Anesthesia & $19(31 \%)$ & $7(41 \%)$ & $12(27 \%)$ & 0.36 \\
\hline & Family medicine & $15(25 \%)$ & $2(12 \%)$ & $13(30 \%)$ & 0.20 \\
\hline & Internal medicine & $23(38 \%)$ & $6(35 \%)$ & $17(39 \%)$ & 1.0 \\
\hline & Medical oncology & $45(74 \%)$ & $16(94 \%)$ & $29(66 \%)$ & 0.03 \\
\hline & Nursing & $15(25 \%)$ & $6(35 \%)$ & $9(20 \%)$ & 0.32 \\
\hline & Palliative medicine & $31(51 \%)$ & $9(53 \%)$ & $22(50 \%)$ & 1.0 \\
\hline & Pediatrics & $3(5 \%)$ & $2(12 \%)$ & $1(2 \%)$ & 0.19 \\
\hline & Psychiatry & $7(11 \%)$ & $3(18 \%)$ & $4(9 \%)$ & 0.39 \\
\hline & Radiation oncology & $10(16 \%)$ & $6(35 \%)$ & $4(9 \%)$ & 0.02 \\
\hline & Surgery & $5(8 \%)$ & $2(12 \%)$ & $3(7 \%)$ & 0.61 \\
\hline & Other & $3(5 \%)$ & $2(12 \%)$ & $1(2 \%)$ & 1.0 \\
\hline & $>1$ Specialty & $42(69 \%)$ & $13(76 \%)$ & $29(66 \%)$ & 0.54 \\
\hline \multirow[t]{5}{*}{ All programs } & Q8: No. of ward nurses assigned to PC & & & & \\
\hline & 0 & $11(18 \%)$ & $5(29 \%)$ & $6(14 \%)$ & \\
\hline & $1-5$ & $21(34 \%)$ & $6(35 \%)$ & $15(34 \%)$ & \\
\hline & $6-10$ & $15(25 \%)$ & $4(24 \%)$ & $11(25 \%)$ & \\
\hline & $>10^{\mathrm{c}}$ & $14(23 \%)$ & $2(12 \%)$ & $12(27 \%)$ & 0.11 \\
\hline \multirow[t]{3}{*}{ All programs } & Q9: No. of outpatient clinic nurses assigned to PC & & & & \\
\hline & $\leq 5^{\mathrm{d}}$ & $54(90 \%)$ & $15(88 \%)$ & $39(91 \%)$ & \\
\hline & $>5^{\mathrm{d}}$ & $6(10 \%)$ & $2(12 \%)$ & $4(9 \%)$ & 1.0 \\
\hline \multirow[t]{4}{*}{ All programs } & Q10: No. of chaplains assigned to PC & & & & \\
\hline & 0 & $20(34 \%)$ & $5(29 \%)$ & $15(36 \%)$ & \\
\hline & 1 & $27(46 \%)$ & $6(35 \%)$ & $21(50 \%)$ & \\
\hline & $2-5$ & $12(20 \%)$ & $6(35 \%)$ & $6(14 \%)$ & 0.19 \\
\hline \multirow[t]{2}{*}{ All programs } & Q11: No. of dietitians assigned to PC & & & & \\
\hline & 0 & $20(33 \%)$ & $7(44 \%)$ & $13(30 \%)$ & \\
\hline
\end{tabular}


Table 1 (continued)

\begin{tabular}{|c|c|c|c|c|c|}
\hline Group & Question & $\begin{array}{l}\text { All } \\
\text { leaders } \\
\text { Combined }\end{array}$ & $\begin{array}{l}\text { Programs } \\
\leq 5 \text { years } \\
\text { old } \\
(n=17)\end{array}$ & $\begin{array}{l}\text { Programs } \\
>5 \text { years old } \\
(n=44)\end{array}$ & $p^{\mathrm{a}}$ \\
\hline & 1 & $33(55 \%)$ & $8(50 \%)$ & $25(57 \%)$ & \\
\hline & $2-5$ & $7(12 \%)$ & $1(6 \%)$ & $6(14 \%)$ & 0.25 \\
\hline \multirow[t]{5}{*}{ All programs } & Q12: No. of mid-level providers assigned to PC & & & & \\
\hline & 0 & $17(29 \%)$ & $6(40 \%)$ & $11(26 \%)$ & \\
\hline & 1 & $12(21 \%)$ & $4(27 \%)$ & $8(19 \%)$ & \\
\hline & $2-5$ & $20(34 \%)$ & $3(20 \%)$ & $17(40 \%)$ & \\
\hline & $>5^{\mathrm{e}}$ & $9(16 \%)$ & $2(13 \%)$ & $7(16 \%)$ & 0.21 \\
\hline \multirow[t]{4}{*}{ All programs } & Q13: No. of PC physicians assigned to PC & & & & \\
\hline & $0-1^{\mathrm{f}}$ & $8(13 \%)$ & $3(18 \%)$ & $5(12 \%)$ & \\
\hline & $2-5$ & $41(68 \%)$ & $11(65 \%)$ & $30(70 \%)$ & \\
\hline & $>5^{\mathrm{f}}$ & $11(18 \%)$ & $3(18 \%)$ & $8(19 \%)$ & 0.67 \\
\hline \multirow[t]{4}{*}{ All programs } & Q14: No. of rehab. personnel assigned to PC & & & & \\
\hline & 0 & $28(47 \%)$ & $7(44 \%)$ & $21(48 \%)$ & \\
\hline & 1 & $17(28 \%)$ & $5(31 \%)$ & $12(27 \%)$ & \\
\hline & $2-5$ & $15(25 \%)$ & $4(25 \%)$ & $11(25 \%)$ & 0.87 \\
\hline \multirow[t]{3}{*}{ All programs } & Q15: No. of pharmacists assigned to PC & & & & \\
\hline & 0 & $32(54 \%)$ & $9(60 \%)$ & $23(52 \%)$ & \\
\hline & $>0^{\mathrm{g}}$ & $27(46 \%)$ & $6(40 \%)$ & $21(48 \%)$ & 0.77 \\
\hline \multirow[t]{3}{*}{ All programs } & Q16: No. of psychiatrists assigned to PC & & & & \\
\hline & 0 & $32(56 \%)$ & $9(56 \%)$ & $23(56 \%)$ & \\
\hline & 1 & $25(44 \%)$ & $7(44 \%)$ & $18(44 \%)$ & 1.0 \\
\hline \multirow[t]{4}{*}{ All programs } & Q17: No. of psychologists assigned to PC & & & & \\
\hline & 0 & $7(12 \%)$ & $1(6 \%)$ & $6(14 \%)$ & \\
\hline & 1 & $32(54 \%)$ & $8(50 \%)$ & $24(56 \%)$ & \\
\hline & $2-5$ & $20(34 \%)$ & $7(44 \%)$ & $13(30 \%)$ & 0.26 \\
\hline \multirow[t]{4}{*}{ All programs } & Q18: No. of social workers assigned to PC & & & & \\
\hline & 0 & $9(16 \%)$ & $1(6 \%)$ & $8(19 \%)$ & \\
\hline & 1 & $30(52 \%)$ & $10(63 \%)$ & $20(48 \%)$ & \\
\hline & $>1^{\mathrm{h}}$ & $19(33 \%)$ & $5(31 \%)$ & $14(33 \%)$ & 0.59 \\
\hline \multirow[t]{4}{*}{ All programs } & Q19: No. of other personnel assigned to PC & & & & \\
\hline & 0 & $20(34 \%)$ & $5(31 \%)$ & $15(36 \%)$ & \\
\hline & 1 & $12(21 \%)$ & $5(31 \%)$ & $7(17 \%)$ & \\
\hline & $>1^{\mathrm{i}}$ & $26(45 \%)$ & $6(38 \%)$ & $20(48 \%)$ & 0.83 \\
\hline \multirow[t]{2}{*}{ All programs } & Q20: FTE physician positions available to PC & & & & \\
\hline & Median (range) & $2(0-15)$ & $2(0-15)$ & $2(0-14)$ & 0.33 \\
\hline \multirow[t]{5}{*}{ All programs } & Q21: No. of physicians with $\geq 20 \%$ protected time & & & & \\
\hline & 0 & $27(45 \%)$ & $10(63 \%)$ & $17(39 \%)$ & \\
\hline & 1 & $12(20 \%)$ & $3(19 \%)$ & $9(20 \%)$ & \\
\hline & $2-3$ & $10(17 \%)$ & $1(6 \%)$ & $9(20 \%)$ & \\
\hline & $4-9$ & $11(18 \%)$ & $2(12 \%)$ & $9(20 \%)$ & 0.11 \\
\hline \multirow[t]{3}{*}{ All programs } & Q22: M.D. certification (finished a fellowship) required? & & & & \\
\hline & No & $25(42 \%)$ & $4(25 \%)$ & $21(48 \%)$ & \\
\hline & Yes & $35(58 \%)$ & $12(75 \%)$ & $21(52 \%)$ & 0.15 \\
\hline \multirow[t]{2}{*}{ All programs } & Q23: Nursing certification (taken boards) required? & & & & \\
\hline & No & $28(47 \%)$ & $5(31 \%)$ & $23(52 \%)$ & \\
\hline
\end{tabular}


Table 1 (continued)

\begin{tabular}{|c|c|c|c|c|c|}
\hline Group & Question & $\begin{array}{l}\text { All } \\
\text { leaders } \\
\text { Combined }\end{array}$ & $\begin{array}{l}\text { Programs } \\
\leq 5 \text { years } \\
\text { old } \\
(n=17)\end{array}$ & $\begin{array}{l}\text { Programs } \\
>5 \text { years old } \\
(n=44)\end{array}$ & $p^{\mathrm{a}}$ \\
\hline & Yes & $32(53 \%)$ & $11(69 \%)$ & $21(48 \%)$ & 0.24 \\
\hline \multirow[t]{3}{*}{ All programs } & Q24: How long does the PC team follow patients? & & & & \\
\hline & $\leq 4$ weeks ${ }^{\mathrm{j}}$ & $15(25 \%)$ & $3(19 \%)$ & $12(27 \%)$ & \\
\hline & $\begin{array}{l}>4 \text { weeks (includes "throughout the course } \\
\text { of the patient's disease) }\end{array}$ & $45(75 \%)$ & $13(81 \%)$ & $32(73 \%)$ & 0.74 \\
\hline \multirow[t]{3}{*}{ All programs } & Q25: Dedicated acute care beds & & & & \\
\hline & No & $15(26 \%)$ & $6(38 \%)$ & $9(21 \%)$ & \\
\hline & Yes & $43(74 \%)$ & $10(62 \%)$ & $33(79 \%)$ & 0.31 \\
\hline \multirow[t]{2}{*}{ Dedicated acute care beds } & Q26: Number of PC beds & & & & \\
\hline & Median (range) & $10(0-43)$ & $9(5-30)$ & $10(0-43)$ & 0.98 \\
\hline \multirow[t]{3}{*}{ Dedicated acute care beds } & Q27: Is there a designated PCU? & & & & \\
\hline & No & $11(26 \%)$ & $3(30 \%)$ & $8(25 \%)$ & \\
\hline & Yes & $31(74 \%)$ & $7(70 \%)$ & $24(75 \%)$ & 1.0 \\
\hline \multirow[t]{3}{*}{ Dedicated acute care beds } & Q28: How long has the inpatient PC service been in place? & & & & \\
\hline & $\leq 5$ years & $13(32 \%)$ & $9(100 \%)$ & $2(6 \%)$ & \\
\hline & $>5$ years & $27(68 \%)$ & $-0-$ & $27(94 \%)$ & - \\
\hline \multirow[t]{2}{*}{ Dedicated acute care beds } & Q29: Inpatient PC discharges per month & & & & \\
\hline & Median (range) & $24(2-250)$ & $19(6-40)$ & $25(2-250)$ & 0.33 \\
\hline \multirow[t]{2}{*}{ Dedicated acute care beds } & Q30: Median stay & & & & \\
\hline & Median (range) & $10(3-98)$ & $14.5(8-98)$ & $9.5(3-96)$ & 0.007 \\
\hline \multirow[t]{2}{*}{ Dedicated acute care beds } & Q31: Median days from admission to death ${ }^{\mathrm{k}}$ & & & & \\
\hline & Median (range) & $10.5(3-55)$ & $15(5-37)$ & $9(3-55)$ & 0.36 \\
\hline \multirow[t]{2}{*}{ Dedicated acute care beds } & Q32: Inpatient PC mortality rate & & & & \\
\hline & Median (range) & $40(2-99)$ & $35(5-93)$ & $43(2-99)$ & 0.69 \\
\hline \multirow[t]{6}{*}{ Dedicated acute care beds } & Q33: Reasons for admission ( $\%$-median/range) & & & & \\
\hline & Death imminent & $10(0-90)$ & $10(1-80)$ & $12(0-90)$ & 0.42 \\
\hline & Acute symptom management & $60(0-90)$ & $50(5-80)$ & $60(0-90)$ & 0.47 \\
\hline & Psychosocial reasons & $10(0-50)$ & $30(0-40)$ & $7.5(0-50)$ & 0.35 \\
\hline & Respite & $5(0-40)$ & $10(0-40)$ & $5(0-10)$ & 0.29 \\
\hline & Other reasons & $10(0-100)$ & $5(0-14)$ & $10(0-100)$ & 0.26 \\
\hline \multirow[t]{8}{*}{ Dedicated acute care beds } & Q34: Referral sources (\%-median/range) & & & & \\
\hline & Intensive care unit & $5(0-30)$ & $5(0-30)$ & $0(0-20)$ & 0.11 \\
\hline & Other inpatient units & $20(0-100)$ & $20(3-100)$ & $20(0-90)$ & 0.91 \\
\hline & Emergency room & $10(0-60)$ & $10(0-50)$ & $10(0-60)$ & 0.79 \\
\hline & Outpatient clinics & $25(0-90)$ & $25(0-79)$ & $22.5(0-90)$ & 0.89 \\
\hline & Other hospitals & $5(0-50)$ & $7.5(0-10)$ & $5(0-50)$ & 1.0 \\
\hline & Hospice & $0(0-50)$ & $0(0-5)$ & $0(0-50)$ & 0.49 \\
\hline & Other & $5(0-70)$ & $0(0-40)$ & $15(0-70)$ & 0.44 \\
\hline \multirow[t]{3}{*}{ Dedicated acute care beds } & Q35: \% of patients that receive psychosocial assessments & & & & \\
\hline & $\leq 75 \%$ & $19(45 \%)$ & $6(60 \%)$ & $13(41 \%)$ & \\
\hline & $>75 \%$ & $23(55 \%)$ & $4(40 \%)$ & $19(59 \%)$ & 0.47 \\
\hline \multirow[t]{3}{*}{ Dedicated acute care beds } & Q36: \% of inpatients that have family conferences & & & & \\
\hline & $\leq 75 \% \mathrm{~m}$ & $21(50 \%)$ & $6(60 \%)$ & $15(47 \%)$ & \\
\hline & $>75 \%$ & $21(50 \%)$ & $4(40 \%)$ & $17(53 \%)$ & 0.72 \\
\hline Dedicated acute care beds & $\begin{array}{l}\text { Q37: How often do oncologists attend family conferences? } \\
<15 \% \text { n,o }\end{array}$ & $19(450 / 0)$ & $220 \%$ & & \\
\hline
\end{tabular}


Table 1 (continued)

\begin{tabular}{|c|c|c|c|c|c|}
\hline Group & Question & $\begin{array}{l}\text { All } \\
\text { leaders } \\
\text { Combined }\end{array}$ & $\begin{array}{l}\text { Programs } \\
\leq 5 \text { years } \\
\text { old } \\
(n=17)\end{array}$ & $\begin{array}{l}\text { Programs } \\
>5 \text { years old } \\
(n=44)\end{array}$ & $p^{\mathrm{a}}$ \\
\hline & $26-75 \%{ }^{\circ}$ & $8(19 \%)$ & $3(30 \%)$ & $5(16 \%)$ & \\
\hline & $>75 \%$ & $15(36 \%)$ & $5(50 \%)$ & $10(31 \%)$ & 0.11 \\
\hline \multirow[t]{3}{*}{ Dedicated acute care beds } & Q38: What $\%$ of patients with DNR orders? & & & & \\
\hline & $\leq 75 \%{ }^{\mathrm{n}, \mathrm{p}}$ & $20(49 \%)$ & $6(60 \%)$ & $14(45 \%)$ & \\
\hline & $>75 \%$ & $21(51 \%)$ & $4(40 \%)$ & $17(55 \%)$ & 0.48 \\
\hline \multirow[t]{3}{*}{ All programs } & Q39: Dedicated consultation service? & & & & \\
\hline & No & $5(8 \%)$ & $2(12 \%)$ & $3(7 \%)$ & \\
\hline & Yes & $54(92 \%)$ & $14(88 \%)$ & $40(93 \%)$ & 0.61 \\
\hline \multirow[t]{3}{*}{ Dedicated consult. service } & Q40: How often is it available? & & & & \\
\hline & $<24 / 7^{\mathrm{q}}$ & $30(57 \%)$ & $8(57 \%)$ & $22(56 \%)$ & \\
\hline & $24 / 7$ & $23(43 \%)$ & $6(43 \%)$ & $17(44 \%)$ & 1.0 \\
\hline \multirow[t]{2}{*}{ Dedicated consult. service } & Q41: No. of referrals/month & & & & \\
\hline & Median (range) & $25(3-400)$ & $15(4-40)$ & $30(3-400)$ & 0.04 \\
\hline \multirow[t]{2}{*}{ Dedicated consult. service } & Q42: No. of days from referral to death ${ }^{\mathrm{r}}$ & & & & \\
\hline & Median (range) & $23(7-97)$ & $15(10-40)$ & $25(7-97)$ & 0.21 \\
\hline \multirow[t]{7}{*}{ Dedicated consult. service } & Q43: Referral sources & & & & \\
\hline & Emergency department & $29(54 \%)$ & $8(57 \%)$ & $21(53 \%)$ & 1.0 \\
\hline & Medical oncology & $51(94 \%)$ & $13(93 \%)$ & $38(95 \%)$ & 1.0 \\
\hline & Surgery & $40(74 \%)$ & $11(79 \%)$ & $29(73 \%)$ & 0.74 \\
\hline & Hematology & $34(63 \%)$ & $7(50 \%)$ & $27(68 \%)$ & 0.34 \\
\hline & Radiation oncology & $42(78 \%)$ & $11(79 \%)$ & $31(78 \%)$ & 1.0 \\
\hline & Other & $23(43 \%)$ & $5(36 \%)$ & $18(45 \%)$ & 0.75 \\
\hline \multirow[t]{5}{*}{ All programs } & Q44: Patients seen in the outpatient setting? & & & & \\
\hline & No & $10(17 \%)$ & $5(31 \%)$ & $5(12 \%)$ & \\
\hline & Yes - dedicated PC outpatient clinic only & $27(46 \%)$ & $4(25 \%)$ & $23(53 \%)$ & \\
\hline & Yes - seen in oncology clinics only & $11(19 \%)$ & $5(31 \%)$ & $6(14 \%)$ & \\
\hline & Both & $11(19 \%)$ & $2(13 \%)$ & $9(21 \%)$ & 0.07 \\
\hline \multirow{2}{*}{$\begin{array}{l}\text { Patients seen in outpatient } \\
\text { setting }\end{array}$} & Q45: How many days/week is the clinic held? & & & & \\
\hline & Median (range) & $5(0.5-7)$ & $5(2-7)$ & $5(0.5-7)$ & 0.87 \\
\hline \multirow{2}{*}{$\begin{array}{l}\text { Patients seen inoutpatient } \\
\text { setting }\end{array}$} & Q46: No. of referrals per month & & & & \\
\hline & Median (range) & $30(3-250)$ & $17.5(3-40)$ & $30(5-250)$ & 0.01 \\
\hline \multirow{2}{*}{$\begin{array}{l}\text { Patients seen in outpatient } \\
\text { setting }\end{array}$} & Q47: No. of days from referral to death ${ }^{\mathrm{s}}$ & & & & \\
\hline & Median (range) & $40(5-365)$ & $40(30-182)$ & $37.5(5-365)$ & 0.58 \\
\hline \multirow{7}{*}{$\begin{array}{l}\text { Patients seen in outpatient } \\
\text { setting }\end{array}$} & Q48: Referral Sources & & & & \\
\hline & Emergency department & $15(31 \%)$ & $4(36 \%)$ & $11(29 \%)$ & 0.72 \\
\hline & Medical oncology & $46(94 \%)$ & $11(100 \%)$ & $35(92 \%)$ & 1.0 \\
\hline & Surgery & $32(65 \%)$ & $8(73 \%)$ & $24(63 \%)$ & 0.73 \\
\hline & Hematology & $29(59 \%)$ & $6(55 \%)$ & $23(61 \%)$ & 0.74 \\
\hline & Radiation oncology & $42(86 \%)$ & $9(82 \%)$ & $33(87 \%)$ & 0.65 \\
\hline & Other & $15(31 \%)$ & $4(36 \%)$ & $11(29 \%)$ & 0.72 \\
\hline \multirow[t]{3}{*}{ All Programs } & Q49: Does your institution operate a hospice & & & & \\
\hline & No & $46(77 \%)$ & $13(81 \%)$ & $33(75 \%)$ & \\
\hline & Yes & $14(23 \%)$ & $3(19 \%)$ & $11(25 \%)$ & 0.74 \\
\hline
\end{tabular}

Programs with a hospice Q50: Average hospice daily census

Median (range)

Only 6 responses 
Table 1 (continued)

\begin{tabular}{|c|c|c|c|c|c|}
\hline Group & Question & $\begin{array}{l}\text { All } \\
\text { leaders } \\
\text { Combined }\end{array}$ & $\begin{array}{l}\text { Programs } \\
\leq 5 \text { years } \\
\text { old } \\
(n=17)\end{array}$ & $\begin{array}{l}\text { Programs } \\
>5 \text { years old } \\
(n=44)\end{array}$ & $p^{\mathrm{a}}$ \\
\hline \multirow[t]{3}{*}{ All programs } & Q51: Palliative medicine Fellowship program? & & & & \\
\hline & No & $36(63 \%)$ & $12(86 \%)$ & $24(56 \%)$ & \\
\hline & Yes & $21(37 \%)$ & $2(14 \%)$ & $19(44 \%)$ & 0.06 \\
\hline \multirow{3}{*}{$\begin{array}{l}\text { Programs with pall. } \\
\text { med. fellowship }\end{array}$} & Q52: No. of clinical fellows/year & & & & \\
\hline & $1-2$ & $11(52 \%)$ & $1(50 \%)$ & $10(53 \%)$ & \\
\hline & $>2^{t}$ & $10(48 \%)$ & $1(50 \%)$ & $9(47 \%)$ & 1.0 \\
\hline \multirow{4}{*}{$\begin{array}{l}\text { Programs with pall. } \\
\text { med. fellowship }\end{array}$} & Q53: No. of research fellows/year & & & & \\
\hline & 0 & $7(44 \%)$ & $-0-$ & $7(50 \%)$ & \\
\hline & 1 & $6(38 \%)$ & $1(50 \%)$ & $5(36 \%)$ & \\
\hline & 2 & $3(19 \%)$ & $1(50 \%)$ & $2(14 \%)$ & 0.14 \\
\hline \multirow[t]{4}{*}{ All programs } & Q54: Mandatory PC rotations for med onc/hem fellows? & & & & \\
\hline & No & $21(36 \%)$ & $5(31 \%)$ & $16(37 \%)$ & \\
\hline & Yes & $27(46 \%)$ & $6(38 \%)$ & $21(49 \%)$ & 0.31 \\
\hline & Not applicable & $11(19 \%)$ & $5(31 \%)$ & $6(14 \%)$ & $1.0^{\mathrm{u}}$ \\
\hline \multirow[t]{4}{*}{ All programs } & Q55: Mandatory PC rotations for radiation onc fellows? & & & & \\
\hline & No & $29(49 \%)$ & $9(56 \%)$ & $20(47 \%)$ & \\
\hline & Yes & $14(24 \%)$ & $1(6 \%)$ & $13(30 \%)$ & 0.14 \\
\hline & Not applicable & $16(27 \%)$ & $6(38 \%)$ & $10(23 \%)$ & $0.13^{\mathrm{u}}$ \\
\hline \multirow[t]{4}{*}{ All programs } & Q56: Mandatory PC rotations for pediatric onc fellows? & & & & \\
\hline & No & $31(54 \%)$ & $9(60 \%)$ & $22(52 \%)$ & \\
\hline & Yes & $3(5 \%)$ & $-0-$ & $3(7 \%)$ & 0.55 \\
\hline & Not applicable & $23(40 \%)$ & $6(40 \%)$ & $17(40 \%)$ & $0.55^{\mathrm{u}}$ \\
\hline \multirow[t]{4}{*}{ All programs } & Q57: Mandatory PC rotations medical students? & & & & \\
\hline & No & $28(49 \%)$ & $6(40 \%)$ & $22(52 \%)$ & \\
\hline & Yes & $15(26 \%)$ & $3(20 \%)$ & $12(29 \%)$ & 0.27 \\
\hline & Not applicable & $14(25 \%)$ & $6(40 \%)$ & $8(19 \%)$ & $1.0^{\mathrm{u}}$ \\
\hline \multirow[t]{4}{*}{ All programs } & Q58: Mandatory PC rotations for other residents/fellows? & & & & \\
\hline & No & $22(38 \%)$ & $8(50 \%)$ & $14(33 \%)$ & \\
\hline & Yes & $23(40 \%)$ & $2(12 \%)$ & $21(50 \%)$ & 0.03 \\
\hline & Not applicable & $13(22 \%)$ & $6(38 \%)$ & $7(17 \%)$ & $0.04^{\mathrm{u}}$ \\
\hline \multirow[t]{3}{*}{ All programs } & Q59: Train mid-level providers in PC? & & & & \\
\hline & No & $22(39 \%)$ & $9(60 \%)$ & $13(32 \%)$ & \\
\hline & Yes & $34(61 \%)$ & $6(40 \%)$ & $28(68 \%)$ & 0.07 \\
\hline \multirow[t]{3}{*}{ All programs } & Q60: PC grand rounds? & & & & \\
\hline & No & $27(47 \%)$ & $8(53 \%)$ & $19(44 \%)$ & \\
\hline & Yes & $31(53 \%)$ & $7(47 \%)$ & $24(56 \%)$ & 0.56 \\
\hline \multirow{4}{*}{$\begin{array}{l}\text { Programs with PC grand } \\
\text { rounds }\end{array}$} & Q61: No of many grand rounds/week? & & & & \\
\hline & 1 & $21(68 \%)$ & & $\begin{array}{c}4(57 \%) 17 \\
(71 \%)\end{array}$ & \\
\hline & 2 & $4(13 \%)$ & $2(29 \%)$ & $2(8 \%)$ & \\
\hline & 3 & $6(19 \%)$ & $1(14 \%)$ & $5(21 \%)$ & 0.84 \\
\hline \multirow[t]{3}{*}{ All programs } & Q62: Length of training for fellows in certification & & & & \\
\hline & $\leq 1$ year ${ }^{\mathrm{V}}$ & $24(67 \%)$ & $8(89 \%)$ & $16(59 \%)$ & \\
\hline & $>1$ year $^{\mathrm{v}}$ & $12(33 \%)$ & $1(11 \%)$ & $11(41 \%)$ & 0.22 \\
\hline \multirow[t]{2}{*}{ All programs } & Q63: PC board examinations? & & & & \\
\hline & No & $35(60 \%)$ & $10(62 \%)$ & $25(60 \%)$ & \\
\hline
\end{tabular}


Table 1 (continued)

\begin{tabular}{|c|c|c|c|c|c|}
\hline Group & Question & $\begin{array}{l}\text { All } \\
\text { leaders } \\
\text { Combined }\end{array}$ & $\begin{array}{l}\text { Programs } \\
\leq 5 \text { years } \\
\text { old } \\
(n=17)\end{array}$ & $\begin{array}{l}\text { Programs } \\
>5 \text { years old } \\
(n=44)\end{array}$ & $p^{\mathrm{a}}$ \\
\hline & Yes & $23(40 \%)$ & $6(38 \%)$ & $17(40 \%)$ & 1.0 \\
\hline \multirow[t]{3}{*}{ All programs } & Q64: Recognized accreditation requirements & & & & \\
\hline & No & $30(52 \%)$ & $6(40 \%)$ & $24(56 \%)$ & \\
\hline & Yes & $28(48 \%)$ & $9(60 \%)$ & $19(44 \%)$ & 0.37 \\
\hline \multirow[t]{3}{*}{ All programs } & Q65: Is there a research program in $\mathrm{PC}$ & & & & \\
\hline & No & $21(36 \%)$ & $6(40 \%)$ & $15(35 \%)$ & \\
\hline & Yes & $37(64 \%)$ & $9(60 \%)$ & $28(65 \%)$ & 0.76 \\
\hline \multirow[t]{12}{*}{ Programs with research } & Q66: Research team & & & & \\
\hline & Chaplains & $8(22 \%)$ & $1(12 \%)$ & $7(25 \%)$ & 0.65 \\
\hline & Pharmacists & $9(25 \%)$ & $3(38 \%)$ & $6(21 \%)$ & 0.38 \\
\hline & Rehab therapists & $5(14 \%)$ & $1(12 \%)$ & $4(14 \%)$ & 1.0 \\
\hline & Data analysts & $27(75 \%)$ & $7(88 \%)$ & $20(71 \%)$ & 0.65 \\
\hline & Physicians & $36(100 \%)$ & $8(100 \%)$ & $28(100 \%)$ & 1.0 \\
\hline & Research nurses & $26(72 \%)$ & $5(62 \%)$ & $21(75 \%)$ & 0.66 \\
\hline & Dieticians & $6(17 \%)$ & $1(12 \%)$ & $5(18 \%)$ & 1.0 \\
\hline & Psychologists & $20(56 \%)$ & $5(62 \%)$ & $15(54 \%)$ & 0.71 \\
\hline & Social workers & $11(31 \%)$ & $2(25 \%)$ & $9(32 \%)$ & 1.0 \\
\hline & $\begin{array}{l}\text { Full team (physicians, research nurses, data } \\
\text { analysts, psych/soc } \pm \text { others) }\end{array}$ & $16(44 \%)$ & $5(62 \%)$ & $11(39 \%)$ & \\
\hline & $<$ Full team & $20(56 \%)$ & $3(38 \%)$ & $17(61 \%)$ & 0.42 \\
\hline \multirow[t]{3}{*}{ Programs with research } & Q67: Outside funding? & & & & \\
\hline & No & $14(38 \%)$ & $6(67 \%)$ & $8(29 \%)$ & \\
\hline & Yes & $23(62 \%)$ & $3(33 \%)$ & $20(71 \%)$ & 0.06 \\
\hline \multirow{5}{*}{$\begin{array}{l}\text { Programs with research } \\
\text { and funding }\end{array}$} & Q68: Sources of funding & & & & \\
\hline & Private industry & $12(52 \%)$ & $2(67 \%)$ & $10(50 \%)$ & 1.0 \\
\hline & Institutional & $12(52 \%)$ & $-0-$ & $12(60 \%)$ & 0.09 \\
\hline & Peer reviewed & $11(48 \%)$ & $1(33 \%)$ & $10(50 \%)$ & 1.0 \\
\hline & Private foundation/philanthropy & $19(83 \%)$ & $3(100 \%)$ & $16(80 \%)$ & 1.0 \\
\hline \multirow[t]{6}{*}{ Programs with research } & Q69: Types of research & & & & \\
\hline & Prospective studies & $32(86 \%)$ & $7(78 \%)$ & $25(89 \%)$ & 0.58 \\
\hline & Retrospective studies & $21(57 \%)$ & $6(67 \%)$ & $15(54 \%)$ & 0.70 \\
\hline & Case series/reports & $19(51 \%)$ & $2(22 \%)$ & $17(61 \%)$ & 0.06 \\
\hline & Qualitative studies & $20(54 \%)$ & $4(44 \%)$ & $16(57 \%)$ & 0.70 \\
\hline & Other & $2(5 \%)$ & $-0-$ & $2(7 \%)$ & 1.0 \\
\hline \multirow[t]{4}{*}{ Programs with research } & $\begin{array}{l}\text { Q70: No. of abstracts presented at oncology } \\
\text { conferences }\end{array}$ & & & & \\
\hline & 0 & $9(24 \%)$ & $2(22 \%)$ & $7(25 \%)$ & \\
\hline & $1-2$ & $16(43 \%)$ & $3(33 \%)$ & $13(46 \%)$ & \\
\hline & $3-10^{\mathrm{w}}$ & $12(32 \%)$ & $4(44 \%)$ & $8(29 \%)$ & 0.52 \\
\hline \multirow[t]{4}{*}{ Programs with research } & Q71: No. of abstracts presented at PC conferences & & & & \\
\hline & 0 & $13(35 \%)$ & $5(56 \%)$ & $8(29 \%)$ & \\
\hline & $1-2$ & $12(32 \%)$ & $4(44 \%)$ & $8(29 \%)$ & \\
\hline & $>2^{\mathrm{x}}$ & $12(32 \%)$ & $-0-$ & $12(43 \%)$ & 0.03 \\
\hline \multirow[t]{2}{*}{ Programs with research } & Q72: No. of abstracts presented at other meetings & & & & \\
\hline & 0 & $9(25 \%)$ & $2(22 \%)$ & $7(26 \%)$ & \\
\hline
\end{tabular}


Table 1 (continued)

\begin{tabular}{|c|c|c|c|c|c|}
\hline Group & Question & $\begin{array}{l}\text { All } \\
\text { leaders } \\
\text { Combined }\end{array}$ & $\begin{array}{l}\text { Programs } \\
\leq 5 \text { years } \\
\text { old } \\
(n=17)\end{array}$ & $\begin{array}{l}\text { Programs } \\
>5 \text { years old } \\
(n=44)\end{array}$ & $p^{\mathrm{a}}$ \\
\hline & $1-5$ & $23(64 \%)$ & $5(56 \%)$ & $18(67 \%)$ & \\
\hline & $>5^{\mathrm{y}}$ & $4(11 \%)$ & $2(22 \%)$ & $2(7 \%)$ & 0.42 \\
\hline \multirow[t]{4}{*}{ Programs with research } & Q73: No. of papers published in oncology journals & & & & \\
\hline & 0 & $12(32 \%)$ & $4(44 \%)$ & $8(29 \%)$ & \\
\hline & $1-2$ & $11(30 \%)$ & $1(11 \%)$ & $10(36 \%)$ & \\
\hline & $>2^{\mathrm{z}}$ & $14(38 \%)$ & $4(44 \%)$ & $10(36 \%)$ & 0.83 \\
\hline \multirow[t]{4}{*}{ Programs with research } & Q74: No. of papers published in PC journals & & & & \\
\hline & 0 & $11(30 \%)$ & $4(44 \%)$ & $7(25 \%)$ & \\
\hline & $1-2$ & $15(41 \%)$ & $5(56 \%)$ & $10(36 \%)$ & \\
\hline & $>2^{\mathrm{aa}}$ & $11(30 \%)$ & $-0-$ & $11(39 \%)$ & 0.05 \\
\hline \multirow[t]{3}{*}{ Programs with research } & $\begin{array}{l}\text { Q75: No. of papers published in general medical } \\
\text { journals }\end{array}$ & & & & \\
\hline & 0 & $19(51 \%)$ & $2(22 \%)$ & $17(61 \%)$ & \\
\hline & $>0^{\mathrm{bb}}$ & $18(49 \%)$ & $7(78 \%)$ & $11(39 \%)$ & 0.06 \\
\hline \multirow[t]{6}{*}{ Programs with research } & Q76: At what stage do you see cancer patients & & & & \\
\hline & At diagnosis & $8(23 \%)$ & $2(22 \%)$ & $6(23 \%)$ & \\
\hline & During curative therapy & $7(20 \%)$ & $4(44 \%)$ & $3(12 \%)$ & \\
\hline & During palliative therapy & $15(43 \%)$ & $2(22 \%)$ & $13(50 \%)$ & \\
\hline & After anticancer therapy is finished & $2(6 \%)$ & $-0-$ & $2(8 \%)$ & \\
\hline & When actively dying & $3(9 \%)$ & $1(11 \%)$ & $2(8 \%)$ & 0.23 \\
\hline \multirow[t]{6}{*}{ Programs with research } & Q77: Internal meetings PC members attend & & & & \\
\hline & Oncology conferences & $24(65 \%)$ & $6(67 \%)$ & $18(64 \%)$ & 1.0 \\
\hline & Oncology tumor boards & $20(54 \%)$ & $6(67 \%)$ & $14(50 \%)$ & 0.46 \\
\hline & Oncology administrative meetings & $19(51 \%)$ & $4(44 \%)$ & $15(54 \%)$ & 0.71 \\
\hline & Oncology out- or inpatient meetings & $20(54 \%)$ & $4(44 \%)$ & $16(57 \%)$ & 0.70 \\
\hline & All 4 types & $10(27 \%)$ & $2(22 \%)$ & $8(29 \%)$ & 1.0 \\
\hline \multirow[t]{4}{*}{ Programs with research } & $\begin{array}{l}\text { Q78: Do oncology members attend PC meetings/ } \\
\text { conferences? }\end{array}$ & & & & \\
\hline & Palliative medicine lectures & $27(73 \%)$ & $7(78 \%)$ & $20(71 \%)$ & 1.0 \\
\hline & Palliative medicine administrative meetings & $13(35 \%)$ & $3(33 \%)$ & $10(36 \%)$ & 1.0 \\
\hline & Both & $11(30 \%)$ & $2(22 \%)$ & $9(32 \%)$ & 0.69 \\
\hline \multirow[t]{3}{*}{ Programs with research } & Q79: Do oncologists attend PC services? & & & & \\
\hline & No & $11(31 \%)$ & $2(25 \%)$ & $9(33 \%)$ & \\
\hline & Yes & $24(69 \%)$ & $6(75 \%)$ & $18(67 \%)$ & 1.0 \\
\hline \multirow[t]{3}{*}{ Programs with research } & Q80: Do PC specialists attend oncology services? & & & & \\
\hline & No & $11(31 \%)$ & $2(22 \%)$ & $9(35 \%)$ & \\
\hline & Yes & $24(69 \%)$ & $7(78 \%)$ & $17(65 \%)$ & 0.69 \\
\hline \multirow[t]{8}{*}{ Programs with research } & Q81: Is there integration of PC with other departments? & & & & \\
\hline & Intensive care & $11(30 \%)$ & $3(33 \%)$ & $8(29 \%)$ & 1.0 \\
\hline & Geriatrics & $11(30 \%)$ & $2(22 \%)$ & $9(32 \%)$ & 0.69 \\
\hline & Cardiology & $4(11 \%)$ & $1(11 \%)$ & $3(11 \%)$ & 1.0 \\
\hline & Neurology & $8(22 \%)$ & $2(22 \%)$ & $6(21 \%)$ & 1.0 \\
\hline & Pulmonology & $10(27 \%)$ & $2(22 \%)$ & $8(29 \%)$ & 1.0 \\
\hline & Pediatrics & $4(11 \%)$ & $-0-$ & $4(14 \%)$ & 0.55 \\
\hline & Pain medicine & $18(49 \%)$ & $4(44 \%)$ & $14(50 \%)$ & 1.0 \\
\hline
\end{tabular}


Table 1 (continued)

\begin{tabular}{|c|c|c|c|c|c|}
\hline Group & Question & $\begin{array}{l}\text { All } \\
\text { leaders } \\
\text { Combined }\end{array}$ & $\begin{array}{l}\text { Programs } \\
\leq 5 \text { years } \\
\text { old } \\
(n=17)\end{array}$ & $\begin{array}{l}\text { Programs } \\
>5 \text { years old } \\
(n=44)\end{array}$ & $p^{\mathrm{a}}$ \\
\hline & Rehabilitation & $8(22 \%)$ & $3(33 \%)$ & $5(18 \%)$ & 0.37 \\
\hline & Other ${ }^{\mathrm{cc}}$ & $4(11 \%)$ & $-0-$ & $4(14 \%)$ & 0.53 \\
\hline
\end{tabular}

${ }^{\text {a }}$ Fisher's exact test for factors with two levels, chi-square test for unordered categorical factors with $>2$ levels, Cochran-Armitage trend test for ordered categorical factors, Wilcoxon rank sum test for measured factors

${ }^{\mathrm{b}} 1-26, n=19 ; 26-50 \%, n=1$

${ }^{\mathrm{c}} 11-25, n=11 ; 26-50, n=1 ;>50, n=2$

${ }^{\mathrm{d}} 0, n=4 ; 1-5 n=50 ; 6-10, n=5,11-25=1$

e $6-10, n=5 ;>10, n=4$

${ }^{\mathrm{f}} 0, n=1 ; 1, n=7 ; 6-10, n=8 ;>10, n=3$

${ }^{\mathrm{g}} 1, n=23 ; 2-5, n=4$

h $2-5, n=17 ; 6-10, n=2$

i $2-5, n=21 ; 6-10, n=5$

${ }^{\mathrm{j}} 1-7$ days, $n=4 ;>1-4$ weeks, $n=11 ;>1-12$ months, $n=18$, $>2$ years, $n=1$; throughout the course of the patient's illness $-n=26$

${ }^{\mathrm{k}} n=24$

${ }^{1} 0, n=0 ; 1-25 \%, n=4 ; 26-50 \%, n=7 ; 51-75 \%, n=8$

${ }^{\mathrm{m}} 0, n=0 ; 1-25 \%, n=6 ; 26-50 \%, n=6 ; 51-75 \%, n=9$

${ }^{\mathrm{N}}$ survey form says $1-15 \%$, should be $1-25 \%$ ????

${ }^{\circ} 0, n=8 ; 1-15 \%, n=11 ; 26-50, n=5 ; 51-75, n=3$

${ }^{\mathrm{p}} 0, n=1 ; 1-15 \%$ (should be $1-25 \% ? ? ? ?$ ), $n=5 ; 26-50 \%, n=6 ; 51-75 \%, n=8$

q 24 h, weekdays only, $n=3$; business hours/weekdays, $n=24$; other (but not 24/7), $n=3$

${ }^{\mathrm{r}} n=29$

$\mathrm{s}_{n=21}$

${ }^{\mathrm{t}}$ Median (range), 2 (1-22)

${ }^{u}$ Yes vs. no

${ }^{\mathrm{v}}<1$ year, $n=4 ; 2$ years, $n=7 ; 3$ years, $n=4 ; 5$ years, $n=1$

${ }^{\mathrm{w}} 3-5, n=11 ; 6-10, n=1$

${ }^{\mathrm{x}} 3-5, n=8 ; 6-10, n=2 ;>10, n=2$

${ }^{\mathrm{y}} 6-10, n=2 ;>10, n=2$

${ }^{\mathrm{z}} 3-5, n=11 ; 6-10, n=2 ;>10, n=1$

aа $3-5, n=4 ; 6-10, n=3 ;>10, n=4$

${ }^{\mathrm{bb}} 1-2, n=14 ; 3-5, n=3 ; 6-10, n=1$

${ }^{\mathrm{cc}}$ Excludes oncology departments

existence greater than 5 years and has multiple services (inpatient, inpatient consultative, and outpatient). These programs have a limited number of nurses dedicated to palliative care and their leaders have an oncology background plus an addition specialty. Programs usually consist of two full-time physicians who follow patients through the trajectory of illness, manage 10 inpatients, see inpatient and outpatient consults daily, perform a large variety of tasks, and provide teaching in the form of Grand Rounds. A subset will also have fellowship programs with extra responsibilities. Subsets also do research, usually with less than fully staffed research services. More mature programs see more patients, have shorter hospital length of stays and interact with greater frequency with other specialties than oncology. Most programs do publish in peer review journals. This survey did not include administration time, local meetings, and national or international organization responsibilities.

Standardized definitions of palliative care are lacking and the term palliative care has little or no meaning to patients [1]. The use of the term palliative care may put patients off from accepting services as discussed in recent publications from the USA $[9,10]$. Symptom management in this setting is largely supportive.

Different palliative care service models are present throughout Europe. Day care centers are largely present in the UK and hence were excluded from the survey. Palliative 
care inpatient beds per million population range from $>5$ to $>1$ depending on the region or country. The number of palliative care specialists range from 1 per 100,000 to 1 per 1.6 million population [11].

Challenges to palliative care structures and service are prioritization based on both the oncologist and patient perspective. Since the greatest advantage to palliative care services occurs when utilized early in the course of advanced cancer as reported in the USA, outpatient clinics and availability are key [4, 12]. Shared care is primarily achieved through outpatient clinics. Inpatient consultation services and inpatient palliative care units are largely centered on crisis intervention. Multidisciplinary palliative care team values are largely measured by indirect cost savings.

Palliative care as an element of cancer care requires a medical oncologist dedicated to palliative care [13]. The key to success is collaboration, commitment, continuity, communication, and patient centeredness. To achieve this goal, palliative care programs need to be available with multiple service lines in order to provide continuity. Oncologists need to continue to be involved with patients on palliative care units to provide input as to disease course, availability, and appropriateness of further anti-cancer therapy and to communicate with families about goals of care within family conferences.

Palliative care services are underfunded for education and research. Only $1 / 3$ of programs have fellowship training programs. Grant support is largely from private foundations or philanthropic donations rather than governmental sources.

Although there are no universally embraced quality indicators for the organization of palliative care, a number of quality indicators were generated from a Europall project systematic review [14]. These quality indicators include 24/7 availability of the consultative team, a staff that includes a pharmacist, and a single caregiver that can coordinate care to provide continuity across service lines. All team members should be accredited and a psychosocial assessment along with symptom assessment should occur within $24 \mathrm{~h}$ of admission to a palliative care unit. Palliative care programs should have quality assurance, research and fellowship programs [14]. In this regard, many programs fall short in one or more areas.

Educational exchanges between adult and pediatric palliative specialties are very limited. This may be largely due to different cancers and in types of terminal diseases and perhaps different symptom management strategies. The psychosocial stressors and clinical problems are distinctly different. There is probably limited value for pediatric palliative fellows to rotate on adult services.

There are differences between the present study and the survey reported by Dr. Hui [8]. The majority of programs in the present survey have been in existence for 5 years compared with programs surveyed by Dr. Hui (43 of 62, $70 \%$ vs. 39 of 96 or $41 \%$ ). More programs surveyed in the present study followed patients in continuity (4 weeks or greater) than in the study published by Dr. Hui (45 of $62.73 \%$ vs. 26 of $96.27 \%$ ). Half of programs surveyed in both studies required physician certification in palliative medicine. Most leaders of palliative programs in the present study were oncologists with additional specialty training (74\%) whereas in the Hui study only $17 \%$ had an oncology background. The great majority of palliative programs in both surveys had consultative services. However, only a minority provided a $24-\mathrm{h}-7$ day-a-week consultative service. The median time from inpatient consultative referral to death was 23 days in the present study and 7 days in the Hui study. Most programs in the present study had inpatient palliative care beds $(38 / 62,61 \%)$ whereas most programs in the Hui study did not have dedicated beds $(27 / 96$, $28 \%$ ). Median length of stay in hospital was 10 days in both studies. The death rate on the inpatient palliative care units was $40 \%$ in both studies. Dedicated palliative outpatient clinics were available in approximately half of programs reported in both studies. Approximately $20 \%$ of programs had palliative specialists participate in oncology clinics. However, the time from referral to death in the present study was 40 days and in the Hui study 90 days. Palliative care clinics were available 5 days a week in the present study and 2 days a week in the Hui study. Referrals to outpatient palliative care clinics were greater the present study then programs surveyed by Dr. Hui (30 per month vs. 7-14 per month). Less than half of programs had palliative fellowships. Mandatory oncology fellow rotations in palliative care were greater in the programs surveyed in the present study (46\%) than in the Hui study (22-27\%). This was also true of medical student rotations (26\% vs. $12-13 \%$ ). Nearly $2 / 3$ of programs (64\%) in the present study had research as part of the program structure whereas less than half (46\%) of programs in the Hui study were involved with research. The difference between programs may be the result of the ESMO designated palliative programs surveyed in the present study which are required to have a well-developed palliative program integrated into oncology for ESMO recognition. Differences also may reflect differences in palliative care philosophy, service structure and referral between Europe and the USA.

There are several weaknesses of this study. Palliative programs surveyed were largely western European. Responders were likely to be from well-established palliative programs probably embedded in cancer centers. The results would likely to be different if rural and community hospital programs were surveyed. Survey results were influenced by those willing to complete them on line and will likely reflect well-developed programs. We did not query program leaders about financial support of their programs. Questions 76-81 were completed by programs with research structures, though the questions were intended for all completing the survey. This would have skewed the results. The program activity data was largely based on approximate estimates as it is not known how many 
of these programs maintain ongoing quality assurance data to provide more accurate responses.

\section{Conclusions}

Palliative care consists of multiple service lines which provide continuity throughout the course of cancer. Patients are usually seen late in the course of their illness as demonstrated by a high death rate on inpatient palliative care units and short survival in patients seen as outpatients. In general, palliative care is under resourced.

Conflicts of interest The authors have no conflicts of interest.

\section{References}

1. Von Roenn JH, Voltz R, Serrie A (2013) Barriers and approaches to the successful integration of palliative care and oncology practice. $J$ Natl Compr Cancer Netw 11(Suppl 1):S11-S16

2. Von Roenn JH (2013) Optimal cancer care: concurrent oncology and palliative care. J Natl Compr Cancer Netw 11(Suppl 1):S1-S2

3. Smith TJ, Temin S, Alesi ER et al (2012) American Society of Clinical Oncology provisional clinical opinion: the integration of palliative care into standard oncology care. J Clin Oncol Off J Am Soc Clin Oncol 30(8):880-887
4. Temel JS, Greer JA, Muzikansky A et al (2010) Early palliative care for patients with metastatic non-small-cell lung cancer. N Engl J Med 363(8):733-742

5. Abernethy AP, Currow DC (2011) Time for better integration of oncology and palliative care. J Oncol Pract Am Soc Clin Oncol 7(6):346-348

6. Abrahm JL (2012) Integrating palliative care into comprehensive cancer care. J Natl Compr Cancer Netw 10(10):1192-1198

7. Greer JA, Jackson VA, Meier DE, Temel JS (2013) Early integration of palliative care services with standard oncology care for patients with advanced cancer. CA Cancer J Clin 63(5):349-363

8. Hui D, Elsayem A, De la Cruz M et al (2010) Availability and integration of palliative care at US cancer centers. JAMA 303(11): 1054-1061

9. Hui D, De La Cruz M, Mori M et al (2013) Concepts and definitions for "supportive care," "best supportive care," "palliative care," and "hospice care" in the published literature, dictionaries, and textbooks. Support Care Cancer Off J Multinatl Assoc Support Care Cancer 21(3):659-685

10. Dalal S, Palla S, Hui D et al (2011) Association between a name change from palliative to supportive care and the timing of patient referrals at a comprehensive cancer center. Oncologist 16(1):105-111

11. Centeno C, Clark D, Lynch T et al (2007) Facts and indicators on palliative care development in 52 countries of the WHO European region: results of an EAPC task force. Palliat Med 21(6):463-471

12. Dahlin CM, Kelley JM, Jackson VA, Temel JS (2010) Early palliative care for lung cancer: improving quality of life and increasing survival. Int J Palliat Nurs 16(9):420-423

13. Marchetti P, Voltz R, Rubio C, Mayeur D, Kopf A (2013) Provision of palliative care and pain management services for oncology patients. J Natl Compr Cancer Netw 11(Suppl 1):S17-S27

14. Woitha K, Van Beek K, Ahmed N et al (2012) Development of a set of process and structure indicators for palliative care: the Europall project. BMC Health Serv Res 12:381 